\title{
EMBEDDING THEOREMS FOR ASCENDING NILPOTENT GROUPS ${ }^{1}$
}

\section{R. FAUDREE}

In this paper two proofs will be given showing that an ascending nilpotent group can be embedded in the multiplicative group of a division ring, if and only if its subgroup of elements of finite order can be embedded in a division ring. The first proof will depend on the material in a paper by the author (see [3]), and the second proof will use the results in the first part of the paper "On ordered division rings" by B. H. Neumann (see [5]). The construction in the first proof is used to show that any automorphism of the group can be extended to the division ring. First we will determine which countably generated locally finite groups can be embedded in a division ring. As a corollary to this a criterion for embedding locally nilpotent periodic groups will be obtained. With this result we will be able to completely determine which ascending nilpotent groups can be embedded in a division ring.

In this paper a group $G$ will be said to have property $E$ if it can be embedded in a division ring $D$, and to have property $\mathrm{EE}$ if every automorphism of the group $G$ can be extended to be an automorphism of some division ring $D$.

The theorem on countable locally finite groups depends mainly on a result of Amitsur (see [1]). The following notation is adapted from the notation of Amitsur's paper. $\pi$ will denote the set of all primes and $\pi_{1}$ the set of all odd primes $p$ such that 2 has odd order mod $p$. Let $m$ and $r$ be relatively prime integers. Put $s=(r-1, m), t=m / s$ and $n=$ minimal integer satisfying $r^{n} \equiv 1 \bmod m$. Denote by $G_{m, r}$ a group generated by two elements $A$ and $B$ satisfying the relations $A^{m}=1$, $B^{n}=A^{t}$, and $B A B^{-1}=A^{r}$. Denote by $G_{m, r}^{\infty}$ a group $G$ which has an ascending tower of subgroups $\left\{H_{i} \mid 0 \leqq i<\infty\right\}$ such that $G=\bigcup_{i=1}^{\infty} H_{i}$ and each $H_{i}$ is isomorphic to $G_{m_{i}, r_{i}}$ for some relatively prime integers $m_{i}$ and $r_{i}$. Let $s_{i}=\left(r_{i}-1, m_{i}\right), t_{i}=m_{i} / s_{i}$ and $n_{i}=$ minimal in teger satisfying $r_{i}^{n_{i}} \equiv 1 \bmod n_{i} . T^{*}, O^{*}$ and $I^{*}$ will denote the binary tetrahedral, octahedral, and icosahedral groups (see [1]).

Amitsur proved the following:

Theorem 1. A finite group $G$ has property $\mathrm{E}$ if and only if $G$ is isomorphic to one of the following groups:

Presented to the Society, August 30,1966; received by the editors February 14, 1966.

1 Research supported by National Science Foundation grant GP-3990. 
(1) Cyclic group,

(2) $G_{m, r}$ where $m$ and $r$ satisfy condition $C$,

(3) a direct product of $T^{*}$ and $G_{m, r}$ where $G_{m, r}$ is cyclic of order $m$ or of the preceding type, $\left(6,\left|G_{m, r}\right|\right)=1$, and $p \mid m$ implies $p$ in $\pi_{1}$,

(4) $O^{*}$ and $I^{*}$.

Additional notation must be given before condition $C$ can be stated. Let $p$ be a fixed prime dividing $m$.

$\alpha=\alpha_{p}$ is the highest power of $p$ dividing $m$.

$\eta_{p}$ is the minimal integer satisfying $r^{\eta_{p}} \equiv 1 \bmod \left(m p^{-\alpha}\right)$.

$\mu_{p}$ is the minimal integer satisfying $r^{\mu_{p}} \equiv p^{\mu^{\prime}} \bmod \left(m p^{-\alpha}\right)$ for some integer $\mu^{\prime}$.

$\delta_{p}$ is the minimal integer such that $p^{\delta_{p}} \equiv 1 \bmod \left(m p^{-\alpha}\right)$.

$\delta_{p}^{\prime}=\mu_{p} \delta_{p} / \eta_{p}$.

Condition $C$. Integers $m$ and $r$ satisfy condition $C$ if either

(I) $(n, t)=(s, t)=1$ or

(II) $n=2 n^{\prime}, m=2^{\alpha} m^{\prime}, s=2 s^{\prime}$ where $\alpha \geqq 2, m^{\prime}, s^{\prime}$ and $n^{\prime}$ are odd in tegers; $(n, t)=(s, t)=2$ and $r \equiv-1 \bmod 2^{\alpha}$. And either

(III) $n=s=2$ and $r \equiv-1(\bmod m)$ or

(IV) For every $q \mid n$ there exists a prime $p \mid m$ such that $q \nmid \eta_{p}$ and that either

(1) $p \neq 2$ and $\left(q,\left(p^{\delta_{p}^{\prime}}-1\right) / s\right)=1$ or

(2) $p=q=2$, (II) holds and $n / 4 \equiv \delta_{2}^{\prime} \equiv 1(\bmod 2)$.

A generalization of Amitsur's Theorem is:

TheоReм 2. A countably generated locally finite group $G$ has property $E$ if and only if $G$ is isomorphic to one of the following groups:

(1) A subgroup of $\prod_{n \in \pi} Z\left(p^{\infty}\right)$,

(2) $G_{m, r}^{\infty}$ where $m_{i}$ and $r_{i}$ satisfy condition $\mathrm{C}$ for all $i$,

(3) $T^{*} \times H$ where either

(a) $H$ is a subgroup of $\prod_{p \in \pi_{1}} \boldsymbol{Z}\left(p^{\infty}\right)$

(b) $H=G_{m, r}^{\infty}$ where $\left(\left|G_{m_{2}, r_{i}}\right|, 6\right)=1, p \mid m_{i}$ implies $p$ is in $\pi_{1}$ and $m_{i}$ and $r_{i}$ satisfy condition $\mathrm{C}$,

(4) $O^{*}$ and $I^{*}$.

Necessity. If $G$ has a subgroup $H$ which is isomorphic to $I^{*}$, then for any $a$ in $G, G p(H, a)$ is a nonsolvable finite group with property $\mathrm{E}$. Thus $G p(H, a)$ is isomorphic to $I^{*}$ and $G=H$. Similarly if $G$ contains a subgroup $H$ isomorphic to $O^{*}$, then $G$ is isomorphic to $O^{*}$.

If $G$ is not isomorphic to $I^{*}$ or $O^{*}$ and contains a subgroup $H$ which is isomorphic to $T^{*}$, then $G$ contains at most one subgroup isomorphic to $T^{*}$ by (3) of Theorem 1 . Let $\left\{a_{i} \mid 1 \leqq i<\infty\right\}$ be a set of generators of $G$. Then $G_{n}=G p\left(H, a_{1}, \cdots, a_{n}\right)$ is a finite solvable group of class 3 
with property E, hence $G_{n}=H \times K_{n}$ is a group of type (3) of Theorem 1. Thus $G=H \times K$ where $K=\bigcup_{n=1}^{\infty} K_{n}$. If each $K_{n}$ is cyclic, then $K$ is a group satisfying (3a) of Theorem 2. If $K_{n}$ is not cyclic for some $n$, then $K_{m}$ is a group of type (2) of Theorem 1 for all $m \geqq n$; hence $K=\bigcup_{m>n}^{\infty} K_{m}$ is a group of type (3b) of Theorem 2 .

If $G$ has no subgroups isomorphic to $T^{*}, O^{*}$ or $I^{*}$ and $\left\{a_{i} \mid 1 \leqq i<\infty\right\}$ is a set of generators of $G$, then $G_{n}=G p\left(a_{1}, \cdots, a_{n}\right)$ is a group of type (1) or (2) of Theorem 1. Obviously if each $G_{n}$ is of type (1), then $G=\cup_{n=1}^{\infty} G_{n}$ is of type (1) of Theorem 2 ; if one $G_{n}$ is not cyclic, then $G$ is of type (2) of Theorem 2.

Before proving sufficiency, two lemmas will be proved.

LEMмa 1. Let $H_{1} \subseteq H_{2}$ be solvable nonabelian finite groups with property E. If $\sigma_{1}$ is an embedding of $H_{1}$ into a division ring $D_{1}$ such that $\sigma_{1}\left(H_{1}\right)$ generates $D_{1}$, then there is a division ring $D_{2} \supseteq D_{1}$ and an embedding $\sigma_{2}$ of $H_{2}$ into $D_{2}$ such that $\sigma \mid H_{2}=\sigma_{1}$.

Proof. Let $\sigma$ be an embedding of $H_{2}$ in to a division ring $D$ such that $\sigma\left(H_{2}\right)$ generates $D . \sigma\left(H_{1}\right)$ generates a subdivision ring $D^{\prime}$ of $D$. There is an isomorphism $\theta$ from $D_{1}$ onto $D^{\prime}$ such that $\sigma \mid H_{1}=\theta \sigma_{1}$ (see $\left[1\right.$, Lemmas $4,12,13$ and Theorem 6a]). If we identify $D_{1}$ with $D^{\prime}$ using the isomorphism $\theta$, we can assume that $D_{1}=D^{\prime}$ and that $\theta$ is the identity map. Thus if we let $D_{2}=D$ and $\sigma_{2}=\sigma$, then $\sigma_{2} \mid H_{1}=\sigma_{1}$ and $D_{1} \leqq D_{2}$.

LemMA 2. If $G$ is a group with an ascending chain of finite nonabelian subgroups $\left\{H_{i} \mid 1 \leqq i<\infty\right\}$ with property $\mathrm{E}$ such that $\bigcup_{i=1}^{\infty} H_{i}=G$, then $G$ has property EE.

Proof. Using induction and Lemma 1 , it is easily seen that there is a tower of division rings $D_{i}$ and embedding maps $\sigma_{i}$ of $H_{i}$ into $D_{i}(0 \leqq i<\infty)$ such that $\sigma_{i}\left(H_{i}\right)$ generates $D_{i}$ and $\sigma_{i} \mid H=\sigma_{j}$ for all $j \leqq i$. Let $D=\cup_{i=1}^{\infty} D_{i}$. For elements $a$ and $b$ in $D$ there is some $i$ such that $a$ and $b$ are in $D_{i}$. If we define $a+b$ and $a \cdot b$ in $D$ as they are defined in $D_{i}$, then $D$ becomes a division ring. Also the map $\sigma$ of $G$ in to $D$ defined by $\sigma(g)=\sigma_{i}(g)$ if $g$ is in $H_{i}$ is an embedding map of $G$ in to $D$ since $\sigma_{i} \mid H_{j}=\sigma_{j}$ for all $j \leqq i$. Thus $G$ has property E.

There is no loss of generality in assuming that $G$ is actually a subgroup of the multiplicative group of $D$ and that $\sigma$ is the identity map. Let $\theta$ be an automorphism of $G$, and let $D_{i}^{\prime}$ be the subdivision ring of $D$ generated by $\theta\left(H_{i}\right)(0 \leqq i<\infty)$. For each $i$, there is an automorphism $\theta_{i}$ of $D_{i}$ onto $D_{i}^{\prime}$ such that $\theta_{i}\left|H_{i}=\theta\right| H_{i}$ (see [1, Lemmas 4, 12, 13 and Theorem 6a]). $\theta_{i} \mid D_{j}=\theta_{j}$ for $j \leqq i$ since $\theta_{i}\left|H_{j}=\theta_{j}\right| H_{j}$. Hence the map $\theta^{*}$ defined by $\theta^{*}(d)=\theta_{i}(d)$ if $d$ is in $D_{i}$ is an isomorphism of $D$ into $D$ 
extending $\theta$. Since $\theta^{*}(G)=G$ and $G$ generates $D, \theta^{*}$ is an automorphism of $D$. Thus $G$ has property EE.

Now the proof of sufficiency for Theorem 2 can easily be given. Groups of type (4) have property $\mathrm{E}$ by Theorem 1, groups of type (1) have property $\mathrm{E}$ for they are locally cyclic abelian groups (see [2]), and groups of type (2) and (3) have property E by Lemma 2 and Theorem 1.

In a nilpotent group the product of two elements of $p$-power order is also of $p$-power order and elements of relatively prime order commute. Thus if $G$ is a locally nilpotent periodic group, $G \cong \prod_{p \in \pi} G_{p}$ where $G_{p}$ is the subgroup of all elements of $p$-power order. The sylow $p$-subgroup of any finite group with property $E$ is cyclic or isomorphic to the generalized quaternion group $Q_{2^{n}}=G p\left(A, B \mid A^{2^{n}}=1, B^{2}=A^{2^{n-1}}\right.$, $B A B^{-1}=A^{-1}$ ). Thus if $G$ has property $\mathrm{E}, G_{p}$ is an abelian group with property $\mathrm{E}$ for $p \neq 2$ and therefore is countably generated. Hence $G$ is countably generated if $G_{2}$ is countably generated.

If every subgroup of $G_{2}$ is cyclic, then $G_{2}$ is abelian and hence countably generated. If the orders of the subgroups of $G_{2}$ which are generalized quaternion groups are bounded, then $G_{2}$ is a generalized quaternion group. If they are not bounded, $G_{2}$ must have a nonabelian subgroup, hence a generalized quaternion group of arbitrarily high order. Thus $G_{2}$ has a subgroup $H=G p\left(A_{i}, B, 2 \leqq i<\infty \mid A_{i}^{2^{i}}=1\right.$, $A_{i+1}^{2}=A_{i}, B^{2}=A_{2}^{2}$ and $\left.B A_{i} B^{-1}=A_{i}^{-1}\right) . H_{n}=G p\left(A_{n}, B\right)$ is a generalized quaternion group of order $2^{n+1}$. If for $g$ in $G_{2}|g|=2$, then $g=B^{2}$ for a group with property $\mathrm{E}$ has at most one element of order 2 . If $|g|=2^{k}$ for $k>2$, then $g$ is in $\left(A_{k}\right)$ for in a generalized quaternion group there is at most one cyclic group of order $2^{k}$ for $k>2$ and $G p\left(H_{k}, g\right)$ is a generalized quaternion group. If $|g|=4$ then $\left|g B^{-1}\right|=2^{w}$. $w=0$ implies $g=B$, and $w=1$ implies $g B^{-1}=B^{2}$. If $w \geqq 2$, then $G p\left(H_{w}, g\right)$ is a generalized quaternion group say of order $2^{n+1}$. Thus there is an $A$ in $G_{2}$ such that $G p\left(H_{w}, g\right)=G p(A, B)$ with $A^{2^{n}}=1, A^{2^{n-w}}=A_{w}$ and $B A B^{-1}$ $=A^{-1}$. Obviously either $g$ or $g B^{-1}$ is in $(A)$; but since $|g| \leqq\left|g B^{-1}\right|$ $=\left|A_{w}\right|$, either $g$ or $g B^{-1}$ is in $\left(A_{w}\right)$. In either case $g$ is in $H$, thus $G_{2}=H$ and $G_{2}$ is countably generated.

Therefore we have the following:

Corollary. A locally nilpotent periodic group $G$ has property $\mathrm{E}$ if and only if $G$ is isomorphic to:

(1) A subgroup of $\prod_{p \in \pi} Z\left(p^{\infty}\right)$,

(2) $Q_{8} \times H$, where $H$ is a subgroup of $\prod_{p \in \pi} Z\left(p^{\infty}\right)$,

(3) $Q_{2^{n}}$ for $n \geqq 2$,

(4) $Q_{2^{\infty}}=G p\left(A_{i}, B \mid 2 \leqq i<\infty, A_{i}^{2^{i}}=1, A_{i+1}^{2}=A_{i}, B A_{i} B^{-1}=A_{i}^{-1}\right)$. 
Proof. Let $G_{m, r}=G p\left(A, B \mid A^{m}=1, B^{n}=A^{t}\right.$ and $\left.B A B^{-1}=A^{r}\right)$ be a nilpotent group which is not abelian. $G^{(l)}$, the $l$ th member of the lower central series of $G$, is equal to $\left(A^{s l}\right)$. Therefore $G$ is nilpotent if and only if $m \mid s^{l}$. This $G$ does not satisfy I of Condition C and satisfies II of Condition C if and only if $t=2^{\alpha-1}$. Hence $G$ satisfies II and III of Condition $\mathrm{C}$ if and only if $G=G p\left(A, B \mid A^{2^{\alpha}}=1, B A B^{-1}=A^{-1}\right.$ and $\left.B^{2}=A^{2}\right)$, the generalized quaternion group of order $2^{\alpha+1}$.

Assume $G$ satisfies II and IV of Condition C. $r \equiv-1 \bmod 2^{\alpha}$ implies that $r$ has even order $\bmod m / p^{\alpha}$ for any odd prime $p$, hence $G$ must satisfy (2) of IV. Therefore $\alpha=2$ and $t=2$. $t=2$ implies $m \mid 2(r-1)$ or $r \equiv 1 \bmod m / 2$. Thus $r^{2} \equiv 1 \bmod m$ and $n=2$. Obviously $\eta_{2}=\mu_{2}=1$; hence $\delta_{2}^{\prime}=\delta_{2}$, which is the order of $2 \bmod m / 4$. Therefore $G$ satisfies II and IV of Condition C if and only if $n=t=2, s=2 m^{\prime}$ where 2 has odd order modulo the odd integer $m^{\prime}$ and $r \equiv-1 \bmod 4$. Under these conditions $G p(A, B)=\left(A^{4}\right) \times G p\left(A^{m^{\prime}}, B^{m^{\prime}}\right) \cong C_{m} \times Q_{8}$.

Therefore the only finite nilpotent groups with property $\mathrm{E}$ are cyclic groups, generalized quaternion groups or groups isomorphic to $Q_{8} \times C_{m^{\prime}}$, where 2 has odd order $\bmod m^{\prime}$. Since the groups $O^{*}, I^{*}$ and $T^{*}$ are not nilpotent groups, $G$ must be of type (1) or (2) of Theorem 2. Therefore if $G$ is abelian it is of type (1) of the corollary and if it is nonabelian and its sylow 2-subgroup is of order 8 it is of type (2) of the corollary. If the Sylow 2-subgroup is of order $>8$, it is of type (3) or (4) of the corollary.

The periodic elements of a nilpotent group form a fully invariant subgroup (see [6, p. 159]). Since every finitely generated subgroup of an ascending nilpotent group $G$ is a nilpotent group, the periodic elements of an ascending nilpotent group form a fully invariant subgroup, which we will denote by $F_{G}$. We are now prepared to determine which ascending nilpotent groups have property $\mathrm{E}$ and property EE.

THEOREM 3. For an ascending nilpotent group $G$ the following are equivalent:

(1) G has property E,

(2) G has property EE,

(3) $F_{G}$ has property E.

Proof. Obviously (2) implies (1) and (1) implies (3). If $F_{G}$ satisfies (3), then $F_{G}$ has property EE by corollary to Theorem 2, Lemma 2 and [3, Theorem 5]. $G / F_{G}$ has an ascending central series such that each factor group of the series is torsion free (see [6], p. 241). This implies that there is an ascending normal series from $F_{G}$ to $G$ such that each factor group is isomorphic to a subgroup of $R^{+}$, the 
additive rationals. Therefore $G$ has property EE (see [3], Corollary 1 and remark after Corollary 5).

Property $\mathrm{E}$ and property $\mathrm{EE}$ are equivalent for finite groups and abelian groups (see [3]). This along with the above result leads one to ask if these two concepts are equivalent for all groups.

We now give another proof to show that an ascending nilpotent group has property $\mathrm{E}$ if and only if $F_{G}$ has property E. First some definitions will be given.

Let $G$ be an ordered group and let $D$ be a division ring with a set of operators $\Omega .0$ and 1 will denote the additive and multiplicative identities of $D$ respectively. A function $\phi$ defined on $G$ with values in $D$ is called a formal power series if there is a well-ordered subset $S=S(\phi)$ such that $\phi(s) \neq 0$ implies $s$ is in $S$. The set of all formal power series will be denoted by $\Gamma$. A pair of functions $\gamma$ and $\omega$ is called a factor set on $(G, D, \Omega)$ if $\gamma$ is defined on $G \times G$ with values in $D$ and $\omega$ is defined on $G$ with values in $\Omega$ and if they satisfy:

$$
\begin{aligned}
\gamma(r, s) \rho^{\omega(r) \omega(s)} & =\rho^{\omega(r s)} \gamma(r, s), \\
\gamma(r s, t) \gamma(r, s)^{\omega(t)} & =\gamma(r, s t) \gamma(s, t)
\end{aligned}
$$

for all $r, s$ and $t$ in $G$ and $\rho$ in $D$. If $\phi$ and $\chi$ are in $\Gamma$, and if we define addition and multiplication as follows:

$$
\begin{aligned}
& \psi=\phi+\chi, \psi(s)=\phi(s)+\chi(s) \text { for all } s \text { in } G, \\
& \psi=\phi \cdot \chi, \psi(t)=\sum_{r s=t} \gamma(r, s) \phi(r)^{\omega(s)} \chi(s) \text { for all } t \text { in } G,
\end{aligned}
$$

then $\Gamma$ becomes a division ring (see $[\mathbf{5}]$ ).

Let $G$ be an ascending nilpotent group such that $F_{G}$, the subgroup of all periodic elements of $G$, has property E. Thus $F_{G}$ can be embedded in a division ring $D$ such that any automorphism of $G$ can be extended to $D$ by the corollary to Theorem 3. Let $\Omega$ be the set of all automorphisms of $D . G / F_{G}$ is a torsion free ascending nilpotent group and hence can be ordered (see [4]). Since $F_{G}$ is a fully invariant subgroup of $G$ any element $g$ in $G$ induces an automorphism $\sigma_{g}$ of $F_{G}$, namely $\sigma_{\theta}(h)=g^{-1} h g$ for all $h$ in $F_{G}$. Let $\sigma_{g}$ also denote the extension of this automorphism to $D$. Let $T$ be a subset of $G$ which contains exactly one element from each coset of $F_{G}$ in $G$. We can assume that $e$, the identity of $G$, is in $T$. For $g$ in $G$ let $g^{*}$ denote the left coset of $F_{G}$ determined by $g$. We are now prepared to define a factor set for $\left(G / F_{G}, D, \Omega\right)$.

If $r$ and $s$ are in $T$, then $r \cdot s=t h_{r s}$ where $t$ is in $T$ and $h$ is in $F_{G}$. Define $\omega\left(r^{*}\right)=\sigma_{r}$ and $\gamma\left(r^{*}, s^{*}\right)=h_{r s}$. Direct calculation verifies that 
the pair $(\omega, \gamma)$ forms a factor set for $\left(G / F_{G}, D, \Omega\right)$, hence we can form the division ring $\Gamma$ of all formal power series in $G / F_{G}$ over $D$.

Any $g$ in $G$ can be written uniquely in the form $g=t h$ with $t$ in $T$ and $h$ in $F_{g}$. Now consider the map $\theta$ of $G$ into $\Gamma: \theta(g)=\chi_{\theta}$ where $\chi_{\theta}\left(r^{*}\right)=0$ if $r^{*} \neq t^{*}$ and $\chi_{\theta}\left(t^{*}\right)=h$. If $\theta(g)=1$, the multiplicative identity of $\Gamma$, then $\chi_{\theta}\left(e^{*}\right)=1$ and $\chi_{\theta}\left(r^{*}\right)=0$ if $r^{*} \neq e^{*}$. Thus $g=e$ and $\theta$ is one-to-one. Let $g_{i}=t_{i} h_{i}$ with $t_{i}$ in $T$ and $h_{i}$ in $H, i=1$, 2. Then $t_{1} t_{2}$ $=t_{3} \gamma\left(t_{1}^{*}, t_{2}^{*}\right)$ for some $t_{3}$ in $T$.

$$
\chi_{0_{1 \theta_{2}}}=\theta\left(g_{1} g_{2}\right)=\theta\left(t_{1} t_{2} h_{1}^{\omega\left(t_{2}^{*}\right)} h_{2}\right)=\theta\left(t_{3} \gamma\left(t_{1}, t_{2}\right) h_{1}^{\omega\left(t_{2}^{*}\right)} h_{2}\right),
$$

hence

$$
\chi_{\theta_{1} g_{2}}\left(r^{*}\right)=0 \text { if } r^{*} \neq t_{3}^{*}
$$

and

$$
\chi_{0_{102}}\left(t_{3}^{*}\right)=\gamma\left(t_{1}^{*}, \stackrel{t_{2}}{*}\right) h_{1}^{\omega\left(t_{2}\right)} h_{2} .
$$

By the definition of multiplication in $\Gamma$ obviously $\chi_{g_{1}} \chi_{g_{2}}\left(r^{*}\right)=0$ if $r^{*} \neq t_{3}^{*} \quad$ and $\chi_{g_{1}} \chi_{g_{2}}\left(t_{3}^{*}\right)=\gamma\left(t_{1}^{*}, t_{2}^{*}\right) \chi_{\theta_{1}}\left(t_{1}^{*}\right)^{\omega\left(t_{2}^{*}\right)} \chi_{o_{2}}\left(t_{2}^{*}\right)=\gamma\left(t_{1}, t_{2}\right) h_{1}^{\omega}\left(t_{2}^{*}\right) h_{2}$. Thus $\chi_{\theta_{1} \theta_{2}}=\chi_{\theta_{1}} \chi_{\theta_{2}}$ and $\theta$ is an embedding of $G$ into $\Gamma$.

\section{REFERENCES}

1. S. A. Amitsur, Finite subgroups of division rings, Trans. Amer. Math. Soc. 80 (1955), 361-386.

2. P. M. Cohn, Eine Bemerkung über die multiplicative Gruppe eines Körpers, Arch. Math. 13 (1962), 344-348.

3. R. J. Faudree, Subgroups of the multiplicative group of a division ring, Trans. Amer. Math. Soc. 124 (1966), 41-48.

4. K. Iwasawa, On linearly ordered groups, J. Math. Soc. Japan 1 (1948), 1-9.

5. B. H. Neumann, On ordered division rings, Trans. Amer. Math. Soc. 66 (1949), 202-252.

6. H. Zassenhaus, The theory of groups, Chelsea, New York, 1958.

University of California, Berkeley 